\title{
Upbeat nystagmus: clinicoanatomical correlation
}

\author{
J C Janssen, A J Larner, Huw Morris, Adolfo M Bronstein, Simon F Farmer
}

\begin{abstract}
A patient is reported on with isolated upbeating nystagmus with a linear slow phase in whom a solitary lesion, probably inflammatory, was detected radiologically in the dorsal paramedian caudal medulla, encompassing the most caudal of the perihypoglossal nuclei, the nucleus intercalatus of Staderini. The conjunction of a vestibular pattern of nystagmus with this focal lesion runs contrary to a previous suggestion that the nucleus intercalatus may act as a neural integrator for vertical conjugate eye movements.

(F Neurol Neurosurg Psychiatry 1998;65:380-381)
\end{abstract}

Keywords: upbeat nystagmus; clinicoanatomical correlation

"There is still uncertainty about the precise anatomy of coarse upbeat nystagmus". ${ }^{1} \mathrm{Al}-$ though most commonly a reflection of disease at the pontomesencephalic or pontomedullary junctions, upbeat nystagmus has also been reported with lesions of the perihypoglossal and inferior olivary nuclei, brachium conjunctivum, anterior cerebellar vermis, ${ }^{1-4}$ and, on one occasion, more caudal parts of the medulla. ${ }^{5} \mathrm{~A}$ wide range of diseases may be responsible, including infarct, demyelination,

Department of Neurology, St Mary's Hospital, Praed Street, London, UK

J C Janssen

A J Larner

S F Farmer

Neuro-otology Unit, MRC Human

Movement and

Balance Unit, National

Hospital for Neurology and Neurosurgery,

Queen Square,

London, UK

$\mathrm{H}$ Morris

A M Bronstein

Correspondence to:

Dr AJ Larner,

National Hospital for

Neurology and

Neurosurgery, Queen

Square, London,

WC1N 3BG, UK.

Fax 00441718298720 .

Received 31 October 1997 and in revised form 28 January 1998

Accepted 30 January 1998

Different varieties of upbeat nystagmus have been described according to the velocity of the

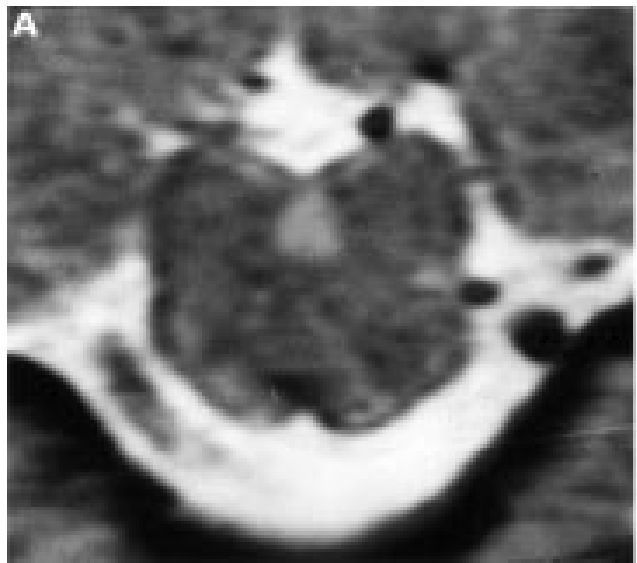

slow phase of the nystagmus-namely, linear or exponentially decaying. Imbalance of vertical vestibulo-ocular reflex activity results in nystagmus with a constant velocity slow phase. ${ }^{2}$ Alternatively, "leakiness" of a neural integrator for vertical conjugate eye movements - a structure which performs a mathematical integration of velocity coded information to position coded information to ensure that the eyes are held steady at the new position after a phasic contraction of the extraocular muscles-may also result in upbeat nystagmus. Damage to the neural integrator results in nystagmus with an exponentially decreasing slow phase velocity. ${ }^{3}$ The neural integrator for horizontal eye movements lies in the nucleus propositus hypoglossi; that for vertical eye movements is thought to reside in the interstitial nucleus of Cajal in the midbrain. The finding of upbeat nystagmus with an exponentially decaying slow phase in a patient with a caudal medullary infarct has prompted the suggestion that vertical integration may be performed partly in the nucleus intercalatus of Staderini, ${ }^{5}$ the most caudal of the perihypoglossal nuclei. ${ }^{6}$ We report on a patient with upbeat nystagmus of vestibular type with a focal lesion of the nucleus intercalatus.

\section{Case report}

A previously well 42 year old right handed TV engineer noted a sudden onset of vertical jumping or bobbing of visual images which he described as "like a disordered vertical hold on

\section{B}

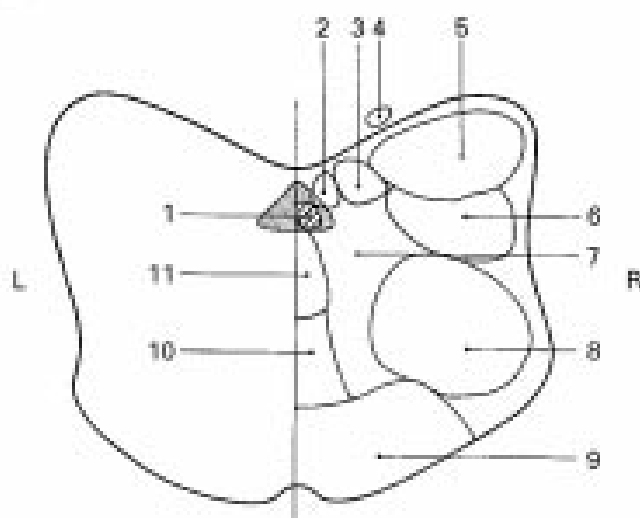

Figure 1 (A) Axial MRI of the caudal brainstem showing focal lesion in dorsal paramedian region. (B) Diagram of brainstem at level of MRI demonstrating anatomical structures: (1) nucleus intercalatus of Staderini (hatched area); (2) dorsal motor nucleus of vagus; (3) nucleus and tractus solitarius; (4) right vertebral artery; (5) cuneate nucleus; (6) nucleus and tract of spinal trigeminal nerve; (7) Internal arcuate fibres; (8) caudal end of inferior olivary nucleus; (9) pyramidal tract; (10) medial lemniscus; (11) medial longitudinal fasciculus. Stippled region corresponds with area of lesion. 


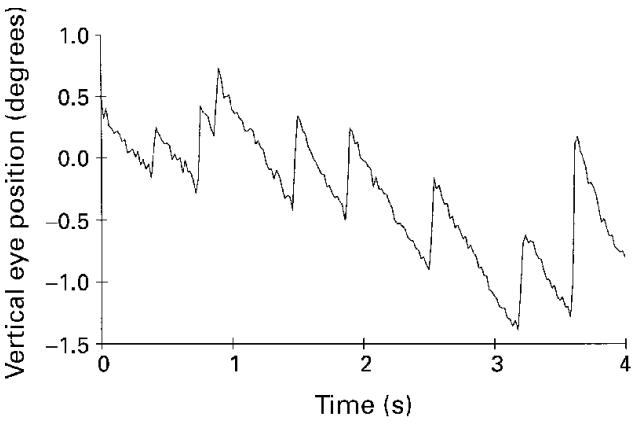

Figure 2 Vertical eye movement recording showing upbeat nystagmus with constant velocity slow phase. Infrared video-oculography; vertical movements of right eye, subject upright and fixating a central spot at $135 \mathrm{~cm}$, full room light.

a TV screen"; he estimated this to be occurring at $4-5 \mathrm{~Hz}$. He could abolish the sensation by laying his head flat on a table, resting his head on either ear, or by standing on his head, but the sensation always returned within a few seconds of returning his head to the vertical. $\mathrm{He}$ thought that his balance was "not right", but he attributed this to his visual problem. There were no other neurological symptoms, and no family history of neurological disease.

His clinical signs were coarse upbeat nystagmus in the primary position, worse on upgaze, with broken vertical pursuit eye movements. The positional manoeuvre (Hallpike and supine or prone position) abolished the nystagmus, coinciding with subjective improvement in symptoms. Neurological and general medical examination was otherwise normal. Over the next 4 weeks the frequency of the oscillopsia gradually declined, and then disappeared.

Investigations two weeks after the onset of oscillopsia included MRI, which showed a single focal lesion in the dorsal paramedian area of the caudal medulla (fig 1A, B). Pure tone audiogram and brainstem auditory evoked potentials were normal. Computerised three dimensional infrared video-oculography (SMI, Berlin) confirmed upbeat nystagmus, the slow phase of which showed a decay of constant velocity in the primary position (fig 2 ) and also with upgaze, when upright or supine, and in the dark. Analysis of CSF showed 11 lymphocytes/ $\mathrm{mm}^{3}$ but protein concentration was normal and oligoclonal bands were absent. Visual evoked potentials from the right eye were delayed (right hemifield latency $120 \mathrm{~ms}$ ). The medullary lesion was not apparent on a repeat MRI two months later, concurrent with the disappearance of the upbeat nystagmus; nor had any new lesions appeared. Repeat visual evoked potentials at this time showed no delay (right hemifield latency $104 \mathrm{~ms}$ ).

\section{Discussion}

Impaired function of the neural integrator, described as "leakiness", results in the eyes drifting back to the primary position at an exponentially decreasing rate, followed by a corrective saccade (gaze paretic nystagmus). The neural integrator for vertical conjugate eye movements is thought to reside in the interstitial nucleus of Cajal in the midbrain, as bilateral lesions of this region lead to a partial failure of vertical gaze holding. The suggestion that the nucleus intercalatus may also play a part in the integration of vertical eye movements rests on the finding of upbeat nystagmus with an exponentially decaying slow phase in one patient with a unilateral lesion of the caudal medulla encompassing that area. ${ }^{5}$ However, this lesion was extensive, also involving the central region of the medulla.

In our patient, upbeat nystagmus was seen in combination with a focal paramedian lesion in the caudal medulla on MRI, encompassing the nucleus intercalatus but not the more central region. The linear slow phase velocity and positional modulation of the nystagmus denotes its vestibular character. The combination of clinical and radiological findings in our patient would therefore argue against the nucleus intercalatus having a role in vertical integration. It should be pointed out, however, that the disappearance of the radiological lesion, concurrent with the clinical signs, does leave some doubt about this interpretation.

The interconnections between the various brainstem regions involved in eye movements are extremely complex, ${ }^{6}$ but there are reports of afferent vestibular input from the descending and medial vestibular nuclei to the nucleus intercalatus. $^{7}$ Hence, a focal lesion of this nucleus could result in a central positional nystagmus, as found with other focal brainstem lesions interrupting central vestibular connections. ${ }^{2}$ Certainly, the marked sensitivity of our patient's nystagmus to head position, as noted by the patient himself and during examination, suggests that the problem was primarily one of input to the ocular motor control systems (vestibular) rather than a primary failure of ocular motol systems.

Thanks are due to Dr Mary Faldon, MRC Human Movement and Balance Unit, National Hospital, Queen Square, for ocular recordings and for preparing fig 2 .

\section{Note added in proof}

Since this paper was accepted, Hirose et al (Ann Neurol 1998;43:403-406) have reported on a patient with upbeat nystagmus in the primary position after a medial medullary infarct. By comparison with other cases of medial medullary infarction without upbeat nystagmus, they conclude that dorsal extension of the lesion to encompass the nucleus intercalatus of Staderini is the critical feature to account for this clinical finding. However, contrary to our findings, they found that the upbeat nystagmus in their patient sometimes had an exponentially decreasing slow phase and hence they concur with the belief of Munro $e t a l^{5}$ that the nucleus intercalatus is a part of the vertical position-to-velocity neural integrator of the human oculomotor system.

1 Adams RD, Victor M, Ropper AH. Principles of neurology. 6th ed. New York: McGraw-Hill, 1997:274.

2 Fisher A, Gresty M, Chambers B, et al. Primary position upbeating nystagmus. A variety of central positional nystagmus. Brain 1983;106:949-64.

3 Brazis PW, Masdeu JC, Biller J. Localization in clinical neurology. 3rd ed. Boston: Little Brown, 1996;208:224-5.

4 Ranalli PJ, Sharpe JA. Upbeat nystagmus and the ventral tegmental pathway of the upward vestibulo-ocular reflex. Neurology 1988;38:1329-30.

5 Munro NAR, Gaymard B, Rivaud S, et al. Upbeat nystagmus in a patient with a small medullary infarct. $\mathcal{F}$ Neurol Neurosurg Psychiatry 1993;56:1126-8.

6 Brodal A. Neurological anatomy in relation to clinical medicine. 3rd ed. Oxford: Oxford University Press, 1981:352-4.

7 Corvaja N, Mergner T, Pompeiano O. Organization of reticular projections to the vestibular nuclei in the cat. Prog Brain Res 1979;50:631-44. 